\section{Orchard Meadow Trees: Tree Detection Using Deep Learning in ArcGIS Pro}

Gl_Forum 2021, Issue 2

Page: 82 - 93

Full Paper

Corresponding Author:

sabine.hennig@sbg.ac.at

DOI: 10.1553/giscience2021_02_s82

\author{
Sabine Hennig \\ Vienna University, Austria
}

\begin{abstract}
'Orchard meadows' refers to the combination of extensively managed fruit trees in combination with fields and pastures. In many regions, among others in Germany, Austria and Switzerland, they are a landscape-defining element and of particular ecological, economic and social importance. However, the numbers of orchard meadows and fruit trees have been decreasing for quite some time. Current and detailed data that allow for the identification of suitable countermeasures to maintain this cultural landscape element are often missing. Such data can be obtained through deep learning. Various deep learning frameworks can now be used in the context of ArcGIS Pro. But what exactly does the use of deep learning involve, in the context of ArcGIS Pro, to get an insight into the stocks of orchard meadow trees? What are the challenges? Initial analyses were carried out using selected areas in Franconian Switzerland (Northern Bavaria) as an example. The results confirm the potential of the approach, but also that training data, model and output data must be refined.
\end{abstract}

\title{
Keywords:
}

cultural landscape, machine learning, monitoring, landscape maintenance

\section{Introduction and research question}

Orchard meadows include half-standard (height $\leq 5 \mathrm{~m}$ ) and standard fruit trees (height $>5 \mathrm{~m}$ and height $\leq 8 \mathrm{~m}$ ), which stand individually, in rows or groups, in meadows, pastures or arable fields, or along paths or ditches, and are extensively cultivated. Depending on the location and cultivation tradition, pome fruits (e.g. apples, pears), stone fruits (e.g. cherries, plums) and shell fruits (walnuts, sweet chestnuts) are grown in orchard meadows. The distances between the individual trees are relatively large to enable the agricultural use of both the fruit trees and the areas under the trees (Haas \& Treter, 1988; Kornprobst, 1994; Prinz et al., 2007).

Due to the occurrence of trees of different ages and different varieties, and the relatively large distances between the individual trees, orchard meadows are a cultural landscape element with high structural diversity. They not only contribute to the characteristic appearance of the corresponding landscape, but also support various aspects of sustainability (Degenbeck, 2004; Girstenbreu, 2006; Haas \& Treter, 1988; Kilian, 2013; Kornprobst, 1994; Prinz et al., 2007). These aspects relate to (i) the natural space: e.g. soil erosion reduction, local climate regulation 
(e.g., temperature, precipitation), supporting high biodiversity; (ii) economic issues: e.g. regional food products (e.g., juice, schnapps), nature-related tourism; (iii) societal aspects: e.g. contributing to nature experience and recreation, importance for education for sustainable development, supporting trends towards more regionality. Orchard meadows are therefore important elements of the cultural landscape. In terms of the functions they fulfil, they cannot be replaced, for example by the dense planting and intensive cultivation of low-growing fruit trees and spindle trees (i.e. by plantations of market fruits) (Haas \& Treter, 1988; Kornprobst, 1994).

Although there are still substantial stocks of orchard meadows in Germany, Austria and Switzerland, their number and size have been in decline since the 1960s. Reasons are the designation of some of the land for building, land consolidation, and conversion into arable land or for large-scale production of market fruits. Tree care and replanting of young trees have often been neglected, so that orchard meadows are now often obsolete, and scrub and forest have encroached on them (Bocheneck, 2019; Degenbeck, 2004; Haas \& Treter, 1988; Kornprobst, 1994; Prinz et al., 2007). In the German state of Bavaria, for example, the number of orchard meadow trees fell by an estimated $70 \%$ from 20 million in 1965 (250,000 ha) to around 5 million in 2013 (approximately 75,000 hectares) (Girstenbreu, 2006; Kilian, 2013). As a result, orchard meadows in Bavaria are now considered an endangered cultural landscape element (LfL, n.d.). Although knowledge of the current situation and the changes taking place are an important basis for the implementation of measures to maintain orchard meadows, there is a lack of detailed and current data regarding orchard meadows and their trees. In Bavaria, such data was last collected in 1965; in 1975, there was an update based on sample counting; later data refer to estimates (Bocheneck, 2019; Degenbeck, 2004; Kilian, 2013).

The collection of data on orchard meadow trees and their monitoring is usually based on onsite mapping (Prinz et al., 2007), but computer-based methods such as deep learning (DL) now offer the possibility of identifying and localizing objects, including trees, in remote sensing images. Moreover, different DL frameworks can also be used in a user-friendly manner in the context of ArcGIS Pro. Using the corresponding geoprocessing tools, which are wellsupported by tutorials, the entire DL workflow can be performed: (i) prepare imagery training data; (ii) train an object detection model; (iii) produce results (Guirado et al., 2017; Paschke, 2020; Schüpferling, 2019). But what exactly does it involve to perform DL in the context of ArcGIS Pro in order to get an insight into the stocks of orchard meadow trees? What are the challenges? These questions were examined in Franconian Switzerland (Northern Bavaria, Germany), where three sites were selected for the provision of training and test data (Figure 1). In this part of the North Franconian Alb, fruit tree cultivation has a long tradition and orchard meadows are a significant part of the cultural landscape (Kornprobst, 1994; Rippel, 2003). 


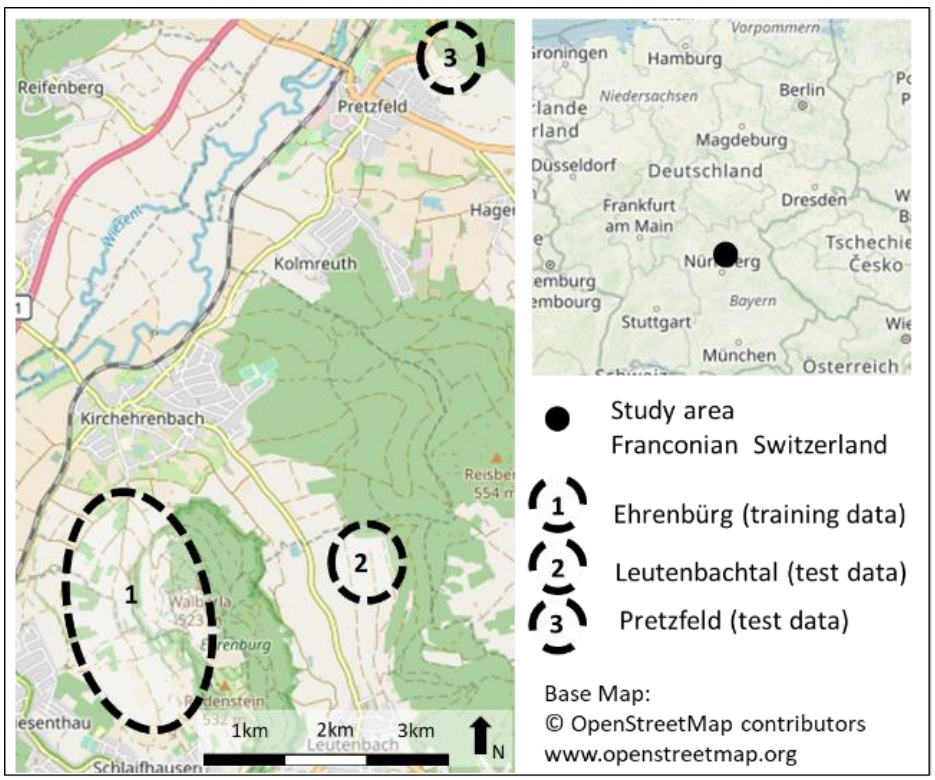

Figure 1: Study area and sites for the provision of training and test data

\section{Workflow, methods and tools}

Deep learning (DL) is a branch of machine learning (ML) and artificial intelligence (AI). It is used, among other things, for image classification and object detection. Using artificial neural networks (ANN) (i.e. models), DL strives to interpret images on the basis of pixel values and recurring patterns and textures, in a manner inspired by biological information processing and the human brain (Goodfellow et al.; 2016; Grekousis, 2019; Sanchez et al., 2020). Briefly, an $\mathrm{ANN}$ is a network of nodes arranged in layers (with weighted connections between them), which receive inputs and produce outputs. ANNs therefore consist of an input layer, an output layer, and one or more layers in between, the so-called hidden layers. ANNs are trained through an iterative process that takes the input data into account. The specific network criteria that are important for correct object detection are defined from the available data; the node connection weights to identify patterns and valuable information are then adjusted. Networks trained in this way can then also be applied to unknown data (Goodfellow et al., 2016; Grekousis, 2019; LeCun et al., 2015).

A special kind of ANN are Convolutional Neural Networks (CNN), introduced by LeCun et al. (1998). CNNs have brought about breakthroughs particularly in image classification and pattern recognition (Grekousis, 2019; LeCun et al., 2015). Their architecture comprises stacks of different operational blocks. The first stacks are convolutional layers. These layers consist of a set of filters (i.e. kernels) that perform scanning operations on the input data and deliver (i) a feature/activation map which keeps the important features, and (ii) pooling layers which, due to down-sampling operations (i.e. applying filters to intensify features), have reduced 
image sizes. The other stacks refer to fully connected layers, i.e. hidden layers, exactly as in typical ANNs (Grekousis, 2019).

As already mentioned, various external DL frameworks can now be used in the context of ArcGIS Pro, with the ArcGIS Image Analyst extension using various geoprocessing tools. The workflow for identifying and locating orchard meadow trees comprises six steps: (i) input image provision; (ii) manual digitization of orchard meadow trees as a basis for the creation of training data, and as test data (i.e., ground truth features) for reviewing the results; (iii) generating training data; (iv) training the network, i.e. creating the model; (v) detecting objects, i.e. orchard meadow trees; (vi) reviewing the results, i.e. computing the accuracy for the orchard meadow trees detected, taking into account the ground truth features.

The work was carried out using ArcGIS Pro 2.7.0. For a visualization of the steps, and details of the geoprocessing tools including input parameters and values used, see Figure 2. As detailed in Figure 2 step 1, the basis for the DL process and all related work is ESRI's World Imagery (for Export) (ESRI, 2019), which (for the study area) was downloaded in ArcGIS Pro and pre-processed.

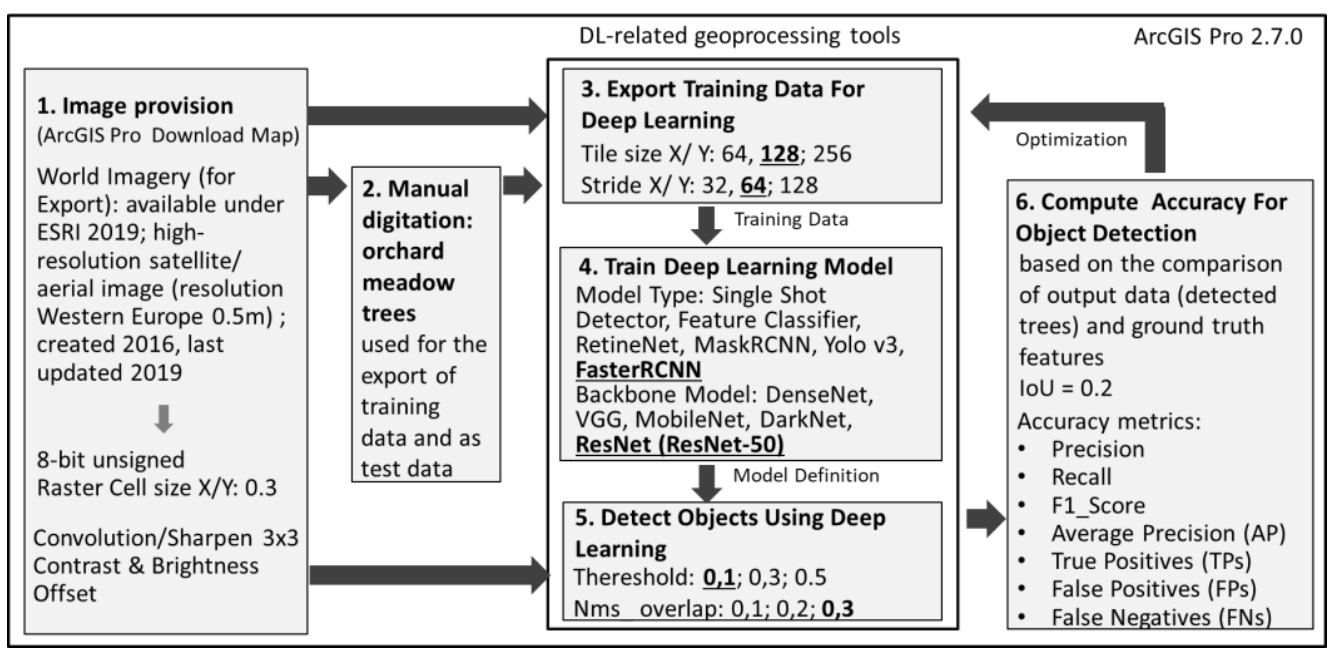

Figure 2: Workflow for the detection of orchard meadow trees using DL in the context of ArcGIS Pro. If no default values have been used, input parameters and values are mentioned. The values include those used for experimental comparison to decide the most suitable values. The values ultimately used, based on the available hardware resources (AMD RyzenTM 7 CPU, 16 GB RAM, 512 GB SSD, Graphics Radeon Vega 10 Mobile), are given in bold and underlined.

In step 2, the orchard meadow trees were digitized manually on-screen using the pre-processed image. This is possible because the distances between the trees are relatively large, and the trees are therefore usually recognizable as individual objects. As a basis for the export of the training data, orchard meadow trees located on the predominantly west-facing slopes of the Ehrenbürg mountain (near the villages of Kirchehrenbach and Schlaifhausen; Figure 1) were selected randomly then digitized (total 1,594 trees, with an average tree canopy base area of $42.2 \mathrm{~m}^{2}$ ). The sites selected as sources for the test data (i.e. trees used for ground truth features) are in 
the Leutenbach valley (Leutenbachtal) and near the village of Pretzfeld (Figure 1). 677 trees (tree canopy base area average: $80.6 \mathrm{~m}^{2}$ ) and 524 trees (tree canopy base area average: $37.3 \mathrm{~m}^{2}$ ) from the Leutenbachtal and Pretzfeld test sites respectively were digitized. Since the ground truth features are used for reviewing and evaluating results (step 6), all trees in both areas were digitized. The manual on-screen digitization of the trees was supported by on-site inspections in autumn 2020.

The corresponding DL-related geoprocessing tools were used for steps 3, 4 and 5. Different input parameters can be selected. Choices for the corresponding values were based (in part) on experimental comparisons. Thus, different values were used for the input parameters (e.g., Tile Size X/Y, Stride X/Y, Threshold, NMS_Overlap) of the tools for Export Training Data For Deep Learning (step 3), and Detect Objects Using Deep Learning (step 5). The results were compared for accuracy (step 6 ). The values ultimately selected, because they gave the best results for the data used, are shown in Figure 2 (bold and underlined).

Findings from the literature were also taken into account in selecting input parameters and values. For Train Deep Learning Model (step 4), various model types (Single Shot Detector, Feature Classifier, RetineNet, MaskRCNN, Yolo v3, FasterRCNN) and backbone models (ResNet, DenseNet, VGG, MobileNet, DarkNet) can be used. They differ in aspects such as application domain, accuracy, computing time, processing capacities, and memory usage (see e.g. Arcos-García et al., 2018; Bianco et al., 2018; Bressem et al., 2018; Chowdhury et al., 2019; Krassimir et al., 2018; Nguyen et al., 2020; Reddy et al., 2018; Ren et al., 2015; Sanchez et al., 2020). Given the available hardware resources (AMD RyzenTM 7 CPU, 16 GB RAM, 512 GB SSD, Graphics Radeon Vega 10 Mobile), using FasterRCNN and ResNet-50 was seen as expedient - a trade-off between accuracy, computing time etc. and the available hardware resources. This approach is in line with, for example, Bressem (2020), Krassimir (2018) and Reddy et al. (2018).

The review and evaluation of the results relied on the Accuracy Computing For Object Detection geoprocessing tool (step 6). Accuracy metrics were computed for:

Precision (the ratio of the number of true positives to the total number of predictions);

Recall (the ratio of the number of true positives to the total number of positive predictions);

F1_Score (The weighted average, with values ranging from 0 to 1 , of the precision and recall combined ; highest accuracy: 1);

Average Precision (AP; the precision averaged across all recall values between 0 and 1);

True Positives (TPs; the number of true positives generated by the model);

False Positives (FP; the number of false positives generated by the model);

False Negatives (FNs; the number of false negatives generated by the model).

The overlap of detected objects and ground truth features is evaluated using the value for Intersection over Union (IoU; Häger et al., 2018; Rezatofighi et al., 2019). Instead of 0.5 as the typical IoU value used, 0.2 was used. This allows inaccuracies in manually digitized (and thus error-prone) ground truth features to be taken into account (Bochinski et al., 2016; Cai \& Vasconcelos, 2018). 
Since the digitized trees are represented by circles (corresponding to the tree canopy base area) but the FasterRCNN model represents detected trees by rectangular bounding boxes, three options for computing the accuracy of the detected trees were considered: (i) digitized trees (circles) and features referring to the largest circle within(?) a rectangular bounding box for the detected trees; (ii) bounding box features representing the manually digitized trees, and rectangular bounding boxes indicating the detected trees; (iii) digitized trees (circles) and rectangular bounding box features representing the detected trees.

\section{Detected orchard meadow trees and their accuracy}

Depending on the input parameter values used, the detected trees and the results of the accuracy metrics vary. The findings presented below are based on the input parameter values that were ultimately used in steps 3, 4 and 5 (Figure 2).

\subsection{Accuracy metrics}

While 524 ground truth features in the Pretzfeld test site were manually mapped, only 257 orchard meadow trees were detected. In the Leutenbachtal test site, 677 ground truth features were mapped and 553 orchard meadow trees were detected.

The accuracy metrics computed with respect to the different options (i.e. bounding boxes/circles) vary only slightly. For this reason, the numbers given in what follows refer to the accuracy metrics computed using bounding boxes for the detected objects, and circles for the ground truth features.

The figures reveal that many trees were not detected, and that some trees were incorrectly identified (Figure 3). For example, Precision is only 0.77 (Pretzfeld) and 0.76 (Leutenbachtal); Recall is 0.38 (Pretzfeld) and 0.62 (Leutenbachtal).
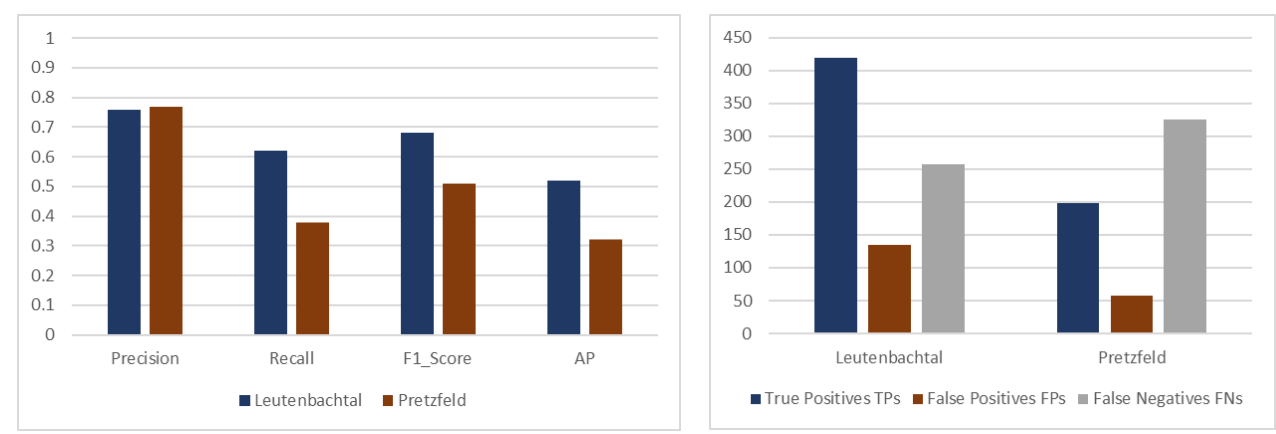

Figure 3: Accuracy metrics for the two test sites, Leutenbach and Pretzfeld (loU=0.2)

The data shows that the trained model works to different degrees in the two test sites, with higher accuracy for Leutenbachtal than for Pretzfeld. In the Leutenbachtal test site, the share of correctly identified trees is $62 \%$ (421 true positive trees detected from 677 ground truth features); in the Pretzfeld site, $38 \%$ of trees were correctly identified (199 out of 525 trees). Of 
the 533 trees in the Leutenbachtal site, 24\% (134 trees) were incorrectly identified. For the Pretzfeld site, the corresponding figure was $23 \%$ (58 out of 257 trees).

\subsection{Undetected and incorrectly detected orchard meadow trees}

In general, orchard meadow trees with a below-average tree canopy base area $\left(50 \mathrm{~m}^{2}\right.$; Kornprobst, 1994) were less well identified than those with a larger canopy base area $(\geq 50$ $\mathrm{m}^{2}$ ). In the Leutenbachtal test site, $49 \%$ of the manually digitized, small-canopy trees were detected, while $76 \%$ of the large-canopy trees were identified; in Pretzfeld, the figures were $36 \%$ of the small-canopy and $63 \%$ of the large-canopy trees (Figure 4 ). This difference between trees with small and large canopy base areas is also highlighted by the examples in Figure 5 (a, b).

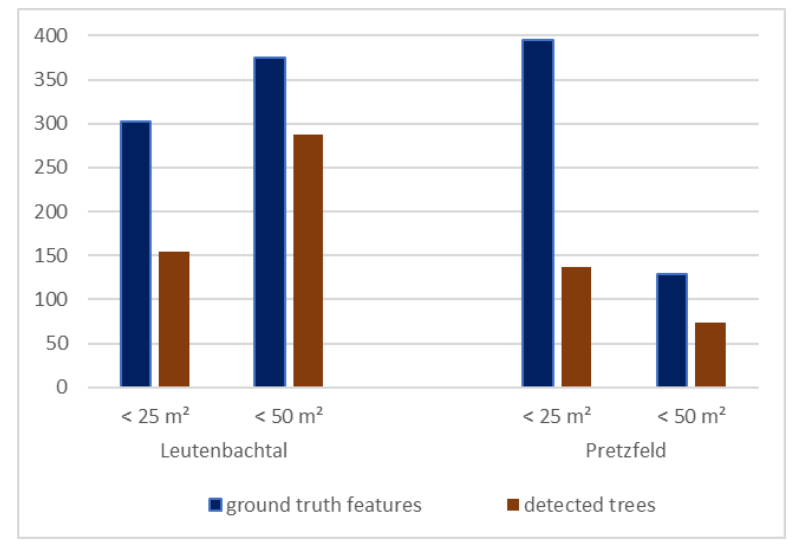

Figure 4: Comparison of ground truth features and orchard meadow trees detected, with regard to different tree canopy base areas, for both test sites

(a)

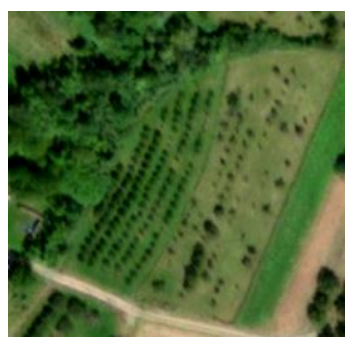

Overview

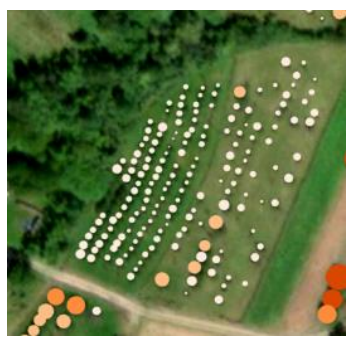

Ground truth features

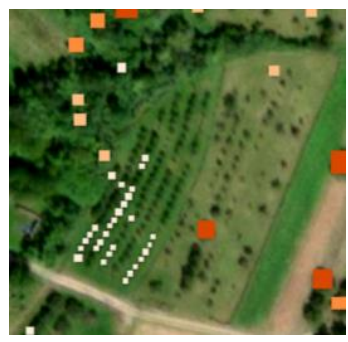

Trees detected 
(b)

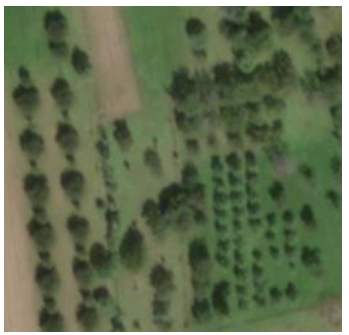

Overview

(c)

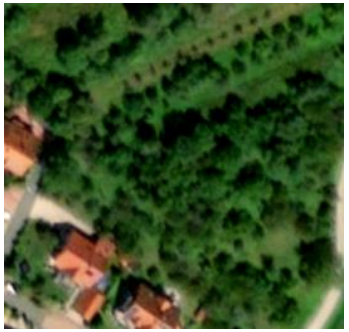

Overview

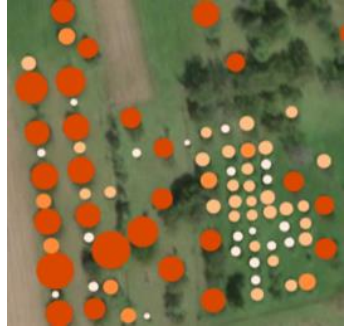

Ground truth features

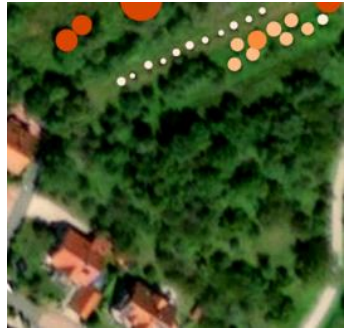

Ground truth features

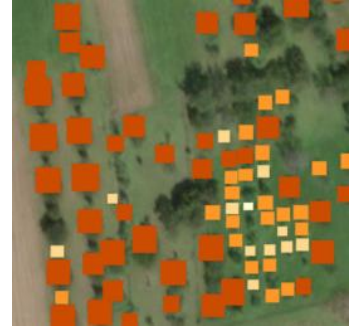

Trees detected

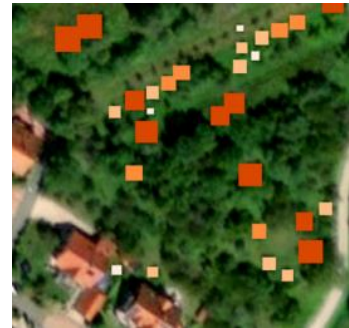

Trees detected

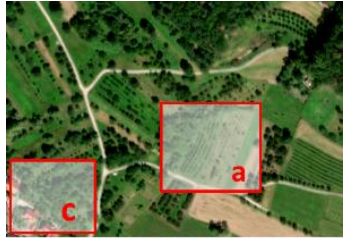

Pretzfeld test site

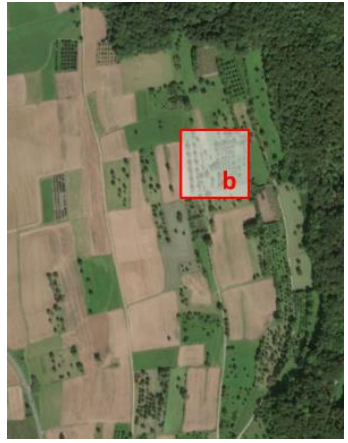

Leutenbachtal test site

\section{Tree canopy base area}

Ground truth features

$$
\begin{aligned}
& <25 \mathrm{~m}^{2} \\
& <50 \mathrm{~m}^{2} \\
& <75 \mathrm{~m}^{2} \\
& >=75 \mathrm{~m}^{2}
\end{aligned}
$$

Detected trees

$$
\begin{aligned}
& <25 \mathrm{~m}^{2} \\
& <50 \mathrm{~m}^{2} \\
& <75 \mathrm{~m}^{2}
\end{aligned}
$$$$
>=75 \mathrm{~m}^{2}
$$

Figure 5: Comparison of trees detected and ground truth features: (a) focus on trees with small canopy base area; (b) focus on trees with large canopy base area; (c) focus on sites encroached by scrub and forest

The difference between small- and large-canopy trees also helps us to understand why in the Leutenbach site the TPs are higher and the FNs are lower than in the Pretzfeld site: the number and share of small-canopy trees in the Leutenbachtal site $(45 \%)$ is considerably lower than in the Pretzfeld site $(75 \%)$.

With regard to the incorrectly detected (FP) orchard meadow trees, the visual analysis shows that these are mainly solitary trees, trees in areas encroached by scrub and forest, or trees in obsolete stocks, at the forest edge or in settlement areas. In such cases, humans were less able to identify the trees when digitizing on-screen, but the trained model did do so (Figure $5 \mathrm{c}$ ). 


\section{Opportunities for result improvement}

The results show that by using ArcGIS Pro DL-related geoprocessing tools for the workflow presented in Figure 2, a first general, but by no means complete, insight into the number and location of orchard meadow trees can be gained. There are several opportunities to improve the model and the results.

\subsection{Training and test data}

For effective model training, suitable and abundant data from which to create training data is required (Bochinski et al., 2016). Thus, the digitization of further orchard meadow trees from which further data are extracted would influence and/or improve the trained model. Moreover, additional on-site inspections would also improve the quality of the training data itself. This would counteract the problem of error, which makes its way into the training data when trees are digitized by humans on-screen (see, e.g., Bochinski et al., 2016). On-site inspections can also increase the quality of ground truth features, and thus have an impact on the accuracy metrics. This refers in particular to situations in which humans did not recognize trees on-screen, but the trained model did. This raises the question of whether trained models perform better than humans (in particular in the case of obsolete stocks of trees, scrub- and forest-encroached orchard meadows, solitary trees), as is also discussed in the literature (see, e.g., He et al., 2015). Related to this, it is essential to clarify whether and to what extent obsolete and scrub- and forest-encroached stocks of fruit trees as well as solitary trees should still be considered orchard meadow trees, taking into account their particular characteristics and multiple functions (as presented in Section 1).

\subsection{Model training}

The decisions on the model type and backbone model to use (FasterRCNN, ResNet-50) were influenced by the available computing power and the models' accuracy. A trade-off between the available hardware resources and the demands of the models in terms of the required computing power is widely accepted, as discussed by Arcos-García et al. (2018) and Sun (2017). Given that other model types and backbone models (e.g., Mask R-CNN; ResNet-101) will result in higher accuracy in object detection, the use of more powerful hardware resources should be considered (Reddy et al., 2018; Krassimir, 2018; Sanchez et al., 2020). In addition, other model configurations and combinations should be used in the context of further experimental comparisons. The creation of training data from other tree canopy base areas (smaller and/or larger) would also be useful.

\subsection{Use of additional data}

False positive objects found in areas that are not suitable for them can be excluded by taking into account data that identifies such areas (Robson et al. 2020). For example, in the mountainous and hilly landscapes of Franconian Switzerland, meadow orchards are located outside settlements, on forest-free, flatter, sun-exposed slopes of Dogger Sandstone (Kornprobst 1994; RvO, 2005). By considering data on land use (e.g. settlements and forest areas), geology, slope inclination and exposure, false positive trees can be excluded. While such 
data is generally available as Open Governmental Data (OGD), for specific natural features crowdsourced data can be valuable in reviewing and/or enriching ground truth feature data and results. For instance, OpenStreetMap (OSM) contains (incomplete) data on features such as orchard meadows, hedges and single trees. To fill the data gaps, citizen science is a promising means of obtaining relevant data from local people and experts (Hennig, 2017; Hennig et al., 2021). Specifically, in the present case, citizen science could help to clarify (for example) whether stocks are obsolete, or if there has been encroachment by scrub and forest.

\section{Conclusion and Outlook}

Orchard meadows are extensively used meadows, pastures or arable land where standard and half-standard fruit trees grow. In many regions in Germany, Austria and Switzerland, they are an important but endangered element of the cultural landscape. Despite their ecological, economic and social importance, the number and extent of orchard meadows have declined in recent decades. For suitable countermeasures to be devised and implemented, current data on orchard meadow trees is needed. DL can be used to identify and locate trees in remote sensing images and fill in the missing data. The use of DL is made easier by the fact that DL frameworks can be used in the context of ArcGIS Pro.

DL-related geoprocessing tools were used to prepare imagery training data, train the object detection model, and produce results. Before and after these particular steps, other tasks had to be carried out using other ArcGIS Pro geoprocessing tools: the imagery had first to be procured, and the digitization of orchard meadow trees had to be carried out manually onscreen. The results also had to reviewed. Although ArcGIS Pro geoprocessing tools open up a well-supported and user-friendly possibility for using DL, particular attention had to be paid to the different input parameters and their values. The example of orchard meadows in Franconian Switzerland (Germany) demonstrates that - on the basis of the available (somewhat limited) hardware resources, which require a trade-off with regard to computing time, processing capacities and accuracy - DL can provide an initial insight into the number and locations of orchard meadow trees. However, in the two test sites, there are significant numbers of false positives or false negatives for the orchard meadow trees detected. This is due to problems detecting trees with small canopy base areas, solitary trees, and trees located in obsolete orchard meadows or meadows encroached on by scrub and forest.

The results presented clearly show that further work is required. Several measures could be taken with the aim of obtaining better results: (i) providing more ground truth features, and supporting the manual on-screen digitization of trees through more on-site inspections; (ii) using other model types and backbone models as well as other input parameter values, even though this would require the availability of better hardware resources; (iii) taking into account data on typical location factors of orchard meadows (e.g. geology, slope, exposure), and relevant natural features (e.g. hedges, single non-fruit trees) to review and/or enrich the data on the digitized trees and the results.

In addition, training data from other areas should be integrated in order to provide an improved and more general model. The appropriately trained model could then be used 
successfully in other regions and thus contribute to the maintenance of this endangered element of the cultural landscape.

\section{References}

Arcos-García, Á., Álvarez-García \& Soria-Morillo, L.M. (2018). Evaluation of deep neuronal networks for traffic sign detection. Neurocomputing 316(2018), 332-344. doi: 10.1016/j.neucom.2018.08.009

Bianco, S., Cadéne, R., Celona, L. \& Napoletano, P. (2018). Benchmark Analysis of Representative Deep Neural Network Architectures. IEEE Access 6. doi: 10.1109/ACCESS.2018.2877890

Bocheneck, J. (2019). Abschlussbericht zum Projekt Inwertsetzung von Streuobstbeständen für eine Modellregion (Mittelfranken). Retrieved from https://www.dbu.de/OPAC/ab/DBUAbschlussbericht-AZ-33535_01-Hauptbericht.pdf

Bochinski, E., Eiselein, V. \& Sikora, T. (2016). Training a convolutional neural network for multi-class object detection using solely virtual world data. 13th IEEE AVSS 2016, 278-285. doi: 10.1109/AVSS.2016.7738056.

Bressem, K., Adams, L., Erxleben, C., Hamm, B., Niehues, S. \& Vahldiek, J. (2020). Comparing different deep learning architectures for classification of chest radiographs. Sci Rep 10(13590). doi: $10.1038 / \mathrm{s} 41598-020-70479-\mathrm{Z}$

Cai, Z. \& Vasconcelos, N. (2018). Cascade R-CNN: Delving into High Quality Object Detection. IEEE/CVF 2018, 6154-6162, doi: 10.1109/CVPR.2018.00644.

Chowdhury D.R., Garg P., More V.N. (2019) Pedestrian Intention Detection Using Faster RCNN and SSD. In M. Singh, P. Gupta, V. Tyagi, J. Flusser, T, Ören \& R. Kashyap (Eds.), Advances in Computing and Data Sciences. ICACDS 2019. Communications in Computer and Information Science, 1046: Springer, Singapore

Degenbeck, K. (2004). Zur Situation der Streuobstbestände in Bayern. Zustand - Probleme Handlungsbedarf. In Bayer. Landesanstalt für Weinbau u. Gartenbau Abt. Landespflege (Eds.), Veitshöchheimer Berichte aus der Landespflege 79/2004.

ESRI Environmental Systems Research Institute (2019). World Imagery (for Export). https://www.arcgis.com/home/item.html?id=226d23f076da478bba4589e7eae95952

Girstenbreu, W. (2006). 5 Jahre Aktion Streuobst 2000 Plus. In Bayer. Landesanstalt für Landwirtschaft (Eds.), Fachtagung Streuobst in der Kulturlandschaft.

Goodfellow, I., Bengio, Y. \& Courvilla, A. (2016). Deep Learning. Retrieved from MIT http://www.deeplearningbook.org

Grekousis, G. (2019). Artificial neural networks and deep learning in urban geography: A systematic review and meta-analysis. Computers, Environment and Urban Systems 74, 244-256. doi: 10.1016/j.compenvurbsys.2018.10.008

Guirado, E., Tabik, S., Alcaraz-Segura, D., Cabello, J. \& Herrera, F. (2017). Deep-learning Versus OBIA for Scattered Shrub Detection with Google Earth Imagery: Ziziphus lotus as Case Study. Remote Sensing 9(12) 1220. doi:10.3390/rs9121220

Haas, D. \& Treter, U. (1988). Die Bedeutung des Streuobstbaus für die Süddeutsche Kulturlandschaft am Beispiel von Wertheim/ Main. FGG Mitteilungen 35(n36), 273-334.

Häger, G., Felsberg, M. \& Khan, F. (2018). Countering bias in tracking evaluations. 13th International Joint Conference on Computer Vision, Imaging and Computer Graphics Theory and Applications - Volume 5: VISAPP, 581-587. doi: 10.5220/0006714805810587

He, K, Zhang, X., Ren, S. \& Sun, J. (2015). Delving Deep into Rectifiers: Surpassing Human-Level Performance on ImageNet Classification. IEEE/ ICCV 2015, 1026-1034. doi: 10.1109/ICCV.2015.123. 
Hennig, S. (2017). OpenStreetMap used in protected area management. The example of the recreational infrastructure in Berchtesgaden National Park. eco.mont 9(2), 30-41. doi: 10.1553/eco.mont-9-2s30

Hennig, S., Abad, L., Hölbling, D. \& Tiede, D. (2021). Implementing Geo Citizen Science Solutions: Experiences from the citizenMorph Project. GI_Fourum Journal 8(1), 2-14. doi: 10.1553/giscience2020_01_s3

Kilian, S. (2013). Bestands- und Bedarfssituation zum Streuobstbau in Bayern aus fachlicher Sicht. In Bayer. Landesanstalt für Landwirtschaft (Eds.), Heimisches Streuobst ist wieder gefragt. Eine Chance für Landwirtschaft und Natur. 11. Kulturlandschaftstag.

Kornprobst, M. (1994). Lebensraumtyp Streuobst. Landschaftspflegekonzept Bayern, Band II.5. StMLU, ANL, München.

Krassimir, V., Schumann, A., Sommer, L. \& Beyerer, J. (2018). A Systematic Evaluation of Recent Deep Learning Architectures for Fine-Grained Vehicle Classification. 29th Pattern Recognition and Tracking (2018). doi: https://doi.org/10.1117/12.2305062

LeCun, Y., Bottou, L., Bengio, Y., Hafner, P. (1998). Gradient-based Learning applied to document recognition. IEEE 86/1, 2278-2324.

LeCun, Y., Bengio, Y. \& Hinton, G. (2015). Deep Learning. Nature 521, 436-444.

LfL Bayer. Landesanstalt für Landwirtschaft (n.d.). Neuanlage und Pflege einer Streuobstwiese. Retrieved from https://www.lfl.bayern.de/iab/kulturlandschaft/142259/index.php

Nguyen, N.D., Do, T., Ngo, T.D. \& Le, D.-D. (2020). An Evaluation of Deep Learning Methods for Small Object Detection. Journal of Electrical and Computer Engineering 20, 1-8. doi: https://doi.org/10.1155/2020/3189691

Paschke, T. (2020). GeoAI: Deep Learning Integration in ArcGIS Pro für Imagery [Blog post] Retrieved from https:/ / arcgis.esri.de/geoai-deep-learning-integration-in-arcgis-pro-fuer-imagery/

Prinz, M., Renetzeder, C, Schmitzberger, I., Stocker-Kiss, A. \& Wrbka, T. (2007). Obstbaumwiesen als Schlüsselelemente zur Erhaltung und Förderung der natürlichen Vielfalt in österreichischen Agrikulturlandschaften. Online-Fachzeitschrift des Bundesministeriums für Land- und Forstwirtschaft, Umwelt und Wasserwirtschaft 2017.

Reddy, N., Rattani, A. \& Derakhshani, R. (2018), Comparison of Deep Learning Models for Biometric-based Mobile User Authentication. IEEE BTAS 2018. doi: 10.1109/BTAS.2018.8698586

Ren, S., He, K., Girshick, R. \& Sun, J. (2015). Faster R-CNN: Towards Real-Time Object Detection with Region Proposal Networks. Computer Vision and Pattern Recognition 2015/1. Retrieved from https://arxiv.org/abs/1506.01497

Rezatofighi, H., Tsoi, N., Gwak, J.Y., Sadeghian, A., Reid, I. \& Savarese, S. (2019). Generalized Intersection over Union: A Metric and A Loss for Bounding Box Regression. IEEE/ CVPR 2019. doi:10.1109/CVPR.2019.00075

Rippel, R. (2003). Aktion „Streuobst 2000 Plus“ - Aus der Region für die Region. In Bayer. Landesanstalt für Landwirtschaft (Eds), Streuobst in der Kulturlandschaft 6/03.

Robson, B.A., Bolch, T., MacDonell, S., Hölbling, D., Rastner, P. \& Schaffer, N. (2020). Automated detection of rock glaciers using deep learning and object-based image analysis. Remote Sensing of Environment 120/2020. doi: https://doi.org/10.1016/j.rse.2020.112033

RvO Regierung von Oberfranken (2005). Landschaftsentwicklungskonzept Region OberfrankenWest, LEK 4.

Sanchez, S.A., Romero, H.J. \& Morales, A.D. (2020). A review: Comparison of performance metrics of pretrained models for object detection using the TensorFlow framework. IOP Conf. Ser.:

Mater. Sci. Eng. 844(2020) 012024. doi: 10.1088/1757-899X/844/1/012024

Schüpferling, R. (2019). Deep Learning mit ArcGIS Pro [Blog post]. Retrieved from https://arcgis.esri.de/deep-learning-mit-arcgis-pro/

Sun, L. (2017). ResNet on Tiny ImageNet. Retrieved from http://cs231n.stanford.edu 